\title{
Desenvolvimento e caracterização de bioplásticos de amido de milho contendo farinha de subproduto de broto
}

\author{
Development and characterization of corn starch \\ bioplastics containing dry sprout by-product flour
}

Maria Luiza Tonetto Silva1 (D), Graziela Brusch Brinques ${ }^{1}$ (D), Poliana Deyse Gurak ${ }^{1 *}$ (D)

${ }^{1}$ Universidade Federal de Ciências da Saúde de Porto Alegre (UFCSPA), Departamento de Nutrição,

Porto Alegre/RS - Brasil

${ }^{*}$ Corresponding Author: Poliana Deyse Gurak, Universidade Federal de Ciências da Saúde de Porto Alegre (UFCSPA), Departamento de Nutrição, Rua Sacramento Leite, 245, CEP: 90050-170, Porto

Alegre/RS, Brasil, e-mail: poligurak@hotmail.com

Cite as: Silva, M. L. T., Brinques, G. B., \& Gurak, P. D. (2020). Development and characterization of corn starch bioplastics containing dry sprout by-product flour. Brazilian Journal of Food Technology, 23, e2018326. https://doi.org/10.1590/1981-6723.32618

\begin{abstract}
Resumo
O objetivo deste trabalho consistiu em desenvolver e caracterizar filmes biodegradáveis de amido de milho contendo farinha de subproduto de broto. Para isso, foi desenvolvida uma farinha a partir de diferentes tipos de broto (feijão, alfafa, amaranto, brócolis, rabanete e, predominantemente, trevo) e realizada sua caracterização tecnológica. Primeiramente, foi produzida farinha de subprodutos de brotos com uso de circulação forçada de ar a $50^{\circ} \mathrm{C}$. As análises físico-químicas demonstraram que a farinha elaborada apresentou coloração marrom-clara, alta capacidade de retenção de água, baixa solubilidade em água e elevado conteúdo de fibras totais. Posteriormente, foram elaboradas soluções filmogênicas contendo amido de milho (4\%), glicerol (30\% sobre o conteúdo de amido de milho) e água destilada (q.s.p. 100\%). Nessas formulações, a farinha de subproduto de broto variou entre 0 (controle), 2,5\%, 5\%, $7,5 \%$ e $10 \%$ em relação ao amido de milho. Os resultados das análises realizadas nos filmes demonstraram que, ao adicionar farinha de subproduto de broto, ocorreu alteração dos parâmetros de cor, no índice de solubilidade em água e na temperatura de fusão, quando comparado o filme controle aos filmes com diferentes proporções de farinha de subproduto de diferentes de brotos. Os valores de solubilidade em ácido e óleo não apresentaram diferenças entre as formulações analisadas. Diante dos resultados obtidos, o subproduto de broto se mostrou como um possível insumo para o desenvolvimento de bioplásticos, visando ao melhoramento das propriedades tecnológicas e ao reaproveitamento dos resíduos agroindustriais.
\end{abstract}

Palavras-chave: Brotos; Subprodutos; Farinha; Bioplásticos; Índice de solubilidade; Temperatura de fusão. 


\begin{abstract}
This study aimed to develop and characterize cornstarch bioplastics containing sprouts by-product flour. Thus, we performed the technological characterization of the sprouts by-product flour of different types of sprouts (beans, alfalfa, amaranth, broccoli, radish and, predominantly, clover). Firstly, we produced sprout by-products flour with forced air circulation at $50^{\circ} \mathrm{C}$. The physicochemical analyses showed that the elaborated flour had high water retention capacity, low water solubility, and high lipid content. After that, we elaborated film-forming solutions with cornstarch (4\%), glycerol (30\% on corn starch content) and distilled water (sufficient quantity to $100 \%$ ). The dry sprout by-product flour was added at proportions of 0 (control); $2.5 \% ; 5 \% ; 7.5 \%$ and $10 \%$ on corn starch base. The results of the films analyzes showed that adding flour of sprouts by-product resulted in changes of the color parameters, water solubility index and melting temperature when comparing the control film to the films with different proportions of the sprouts by-product flour. The acid and oil solubility did not show differences between the analyzed formulations. Our results show that the sprouts by-product proved to be a possible ingredient for improvement of bioplastics, aiming at the improvement of technological properties and the reuse of agroindustrial residues.
\end{abstract}

Keywords: Sprouts; By-products; Flour; Bioplastics; Solubility index; Melting temperature.

\title{
1 Introdução
}

Os plásticos sintéticos são dificilmente substituídos por causa do baixo custo de produção, associado à versatilidade e à resistência. No entanto, o impacto ambiental do uso desses materiais tem incitado o desenvolvimento de substitutos biodegradáveis a partir de fontes naturais renováveis. Embora embalagens de vidro, celulósicas e metálicas sejam bastante empregadas na indústria de alimentos, o plástico é o que tem apresentado o maior crescimento de produção e de emprego como embalagem (Lopez et al., 2018; Karan et al., 2019). Desta forma, há um segmento de mercado consolidado e com perspectivas de crescer, tornando-se um setor lucrativo e aberto a inovações tecnológicas, principalmente com materiais com menor impacto ambiental.

Inúmeras pesquisas têm sido feitas no sentido de incrementar e/ou desenvolver materiais biodegradáveis com características que permitam a sua utilização em embalagens (Rocha et al., 2014; Moro et al., 2017; Lubis et al., 2018; Gomes, et al., 2019; Oluwasina et al., 2019). Os plásticos biodegradáveis, ou bioplásticos, especialmente os provenientes de fontes naturais renováveis, têm sido foco de interesse para o desenvolvimento de novas tecnologias que visam, entre outros aspectos, à preservação ambiental e à busca de potenciais alternativas de substituição de plásticos convencionais oriundos de fontes petrolíferas (Barros et al., 2017).

A elaboração de bioplásticos, como filmes biodegradáveis, inicia-se com a elaboração de uma solução filmogênica, elaborada, basicamente, por três elementos: um agente formador de filme (polissacarídeos, lipídeos e proteínas), um solvente e um plastificante. Cada um desses componentes é utilizado em diferentes combinações, buscando oferecer características distintas para o filme. O bioplástico pode ser utilizado como embalagem ou revestimento aplicado na superfície do alimento e deve apresentar algumas características: propriedades de barreira ao oxigênio, à água e/ou às gorduras, bem como propriedades mecânicas comparáveis aos plásticos convencionais; estabilidade microbiológica, físico-química e bioquímica; ausência de componentes tóxicos ou prejudiciais à saúde, entre outras (Albuquerque \& Malafaia, 2018). Podem também carregar compostos antimicrobianos, antioxidantes e aditivos, além de conferir proteção mecânica e auxiliar no aumento da vida de prateleira dos alimentos (Fakhouri et al., 2012; Melo et al., 2017; Santana et al., 2018). 
Diversos estudos têm sido elaborados utilizando resíduos agroalimentares, visando à conscientização ambiental e ao melhoramento das propriedades físico-químicas, térmicas e mecânicas dos filmes biodegradáveis. Moro et al. (2017), por exemplo, elaboraram uma formulação de bioplástico adicionando até 4\% de casca de maracujá, o que influenciou os parâmetros físicos dos filmes, como uma melhor resistência mecânica, menor permeabilidade ao vapor de água e alongamento intermediário à ruptura. Lubis et al. (2018) desenvolveram bioplásticos de amido de abacate reforçado com celulose microcristalina e determinaram que a formulação que apresentou melhor condição, com valores de $2,74 \mathrm{MPa}$ para resistência à tração e 3,16\% para alongamento na análise de ruptura, foi a com proporção 7:3 de amido por celulose microcristalina com adição de 0,2 (v/w) de glicerol. Portanto, a incorporação de fibras ou outros compostos na formulação pode oferecer efeitos positivos nas características reológicas do bioplástico. Os resíduos dos brotos gerados durante a sua manufatura podem apresentar características oportunas para serem adicionados na formulação de filmes biodegradáveis. Desta forma, o objetivo deste trabalho consistiu em desenvolver e caracterizar filmes biodegradáveis de amido de milho contendo farinha de subproduto de broto.

\section{Material e métodos}

A matéria-prima utilizada foi o resíduo de brotos, gentilmente doado pela empresa Brottar (Viamão, RS, Brasil). Foi recebido um lote com $9 \mathrm{~kg}$ de resíduos (obtidos da produção de broto de feijão, alfafa, amaranto, brócolis, rabanete e, predominantemente, trevo), composto de sementes não germinadas, folhas e brotos avariados in natura. Os brotos que geraram os resíduos desta pesquisa foram produzidos com tecnologia própria em hidroponia, seguindo as boas práticas de produção.

\subsection{Produção das farinhas}

Para produção da farinha, os resíduos de brotos foram secos a $50^{\circ} \mathrm{C}$ com circulação de ar forçada (forno Ltedesco - Vyctory) até peso constante. Após secagem, a amostra foi triturada (mixer Vorwerk) e peneirada (peneira de 60 mesh). Por fim, a farinha de subproduto de broto seco (FSBS) obtida foi pesada e armazenada em embalagens plásticas de polietileno.

\subsection{Elaboração de bioplástico com adição de farinha de subproduto de brotos}

Os equipamentos e os reagentes utilizados para a preparação do filme biodegradável foram: banho-maria (Biomatic -1052-34), banho ultrassônico (Unique - USC-1400), estufa (Ltedesco Vyctory), agitador magnético (Fisatom - modelo 762), amido comercial (maisena - Lote 097), glicerol PA ACS (Alphatec - Lote 22237) e amido solúvel PA ACS (Dinâmica - 83562).

Foram realizadas cinco formulações diferentes de solução filmogênica, sendo os ingredientes o amido de milho, o glicerol, a água destilada e a farinha de subproduto. A fim de comparação, também foi elaborada uma formulação com amido de milho PA, com $0 \%$ de FSBS. As diferentes formulações podem ser observadas na Tabela 1. Para preparação dos filmes biodegradáveis, foi feita uma adaptação da metodologia de Fakhouri et al. (2007) e Crizel et al. (2018). 
Tabela 1. Proporção dos ingredientes utilizados para elaboração dos diferentes filmes biodegradáveis. ${ }^{1}$

\begin{tabular}{lccc}
\hline \multicolumn{1}{c}{ Amostras } & $\begin{array}{c}\text { Amido de milho } \\
\mathbf{( \% )}\end{array}$ & $\begin{array}{c}\text { Glicerol }^{\mathbf{2}, \mathbf{3}} \\
\mathbf{( \% )}\end{array}$ & $\begin{array}{c}\text { FSBS }^{\mathbf{3}} \\
\mathbf{( \% )}\end{array}$ \\
\hline Filmes com 0\% de FSBS com amido PA & 4 & 30 & 0 \\
\hline Filme com 0\% de FSBS (controle) & 4 & 30 & 0 \\
\hline Filme com 2,5\% de FSBS & 4 & 30 & 2,5 \\
\hline Filme com 5\% de FSBS & 4 & 30 & 5 \\
\hline Filme com 7,5\% de FSBS & 4 & 30 & 7,5 \\
\hline Filme com 10\% de FSBS & 4 & 30 & 10 \\
\hline${ }^{1}$ Todas as formulações foram elaboradas com água destilada (q.s.p. 100\%); ${ }^{2}$ Ingredientes invariáveis; ${ }^{3}$ Porcentagem de \\
ingredientes sobre o conteúdo de amido de milho; ${ }^{4}$ Formulação elaborada utilizando amido de milho PA. FSBS - farinha de \\
subproduto de broto seco.
\end{tabular}

A solução filmogênica (SF) foi preparada por meio da pesagem dos componentes escolhidos para cada formulação, mistura dos ingredientes secos (amido de milho e FSBS) e água. Essa mistura foi agitada em agitador magnético por $10 \mathrm{~min}$. Após, o glicerol foi adicionado e aquecido em banho-maria a $85 \pm 1{ }^{\circ} \mathrm{C}$ por 10 min para promover a gelatinização do amido. Na produção do filme de amido PA com $0 \%$ de $\mathrm{FSBS}$, a temperatura foi de $65^{\circ} \mathrm{C} \pm 1{ }^{\circ} \mathrm{C}$ por causa de a temperatura de gelatinização desse amido ser mais baixa. A etapa seguinte foi a secagem dos filmes, na qual foi utilizada a técnica de "casting", definida como a desidratação da solução filmogênica sobre uma placa de Petri. Para tal, foi colocado $0,43 \mathrm{~g}$ de SF por $\mathrm{cm}^{2}$ de área da placa de Petri (Fakhouri et al., 2007). Logo após, foram inseridas as placas em um banho de ultrassom, com o nível de água baixo para evitar a entrada dela, visando retirar possíveis bolhas de ar presente na solução. Em seguida, as placas foram colocadas em uma estufa a $40 \pm 5^{\circ} \mathrm{C}$ por $36 \mathrm{~h}$. Para a remoção efetiva dos filmes da placa de Petri, sem danificar a sua estrutura, eles foram inseridos em um dessecador a temperatura ambiente $\left(25 \pm 1{ }^{\circ} \mathrm{C}\right)$ durante $24 \mathrm{~h}$. Por fim, os filmes foram retirados manualmente das placas de Petri e armazenados em placas de Petri de plástico, as quais foram mantidas dentro de dessecador a temperatura ambiente $\left(25 \pm 1^{\circ} \mathrm{C}\right)$.

\subsection{Análises}

A farinha de subproduto de broto foi caracterizada por meio das análises de umidade, cinzas, proteínas, lipídeos, fibras, carboidrato por diferença, índice de absorção de água, índice de absorção de óleo, índice de solubilidade em água, atividade de água, $\mathrm{pH}$, acidez e cor. Para analisar as propriedades do bioplástico elaborado, foram determinadas umidade, cor, solubilidade em água, solubilidade em ácido, solubilidade em óleo, gramatura, espessura, medida de opacidade e análise das propriedades térmicas.

Análises de umidade, cinzas, proteínas, lipídeos, fibras, carboidratos, acidez e pH seguiram a metodologia descrita pelo Instituto Adolfo Lutz (2008) e pela Association of Official Analytical Chemists (1997). O índice de absorção de água (IAA), o índice de absorção de óleo (IAO) e o índice de solubilidade em água (ISA) foram determinados segundo o método descrito por Guillon \& Champ (2000) e Robertson et al. (2000). A solubilidade em água e a solubilidade em ácido foram analisadas conforme metodologia desenvolvida por Fakhouri et al. (2012). Já a determinação da solubilidade em óleo foi adaptada desse mesmo autor, no qual foi colocada uma seção do biofilme referente a $4 \mathrm{~cm}^{2}$ em estufa a $105^{\circ} \mathrm{C}$ por $24 \mathrm{~h}$; em seguida, a amostra foi pesada e inserida em um béquer com aproximadamente $80 \mathrm{~mL}$ de óleo de soja sob agitação magnética a $25 \pm 1{ }^{\circ} \mathrm{C}$ por $24 \mathrm{~h}$. Logo após, foi retirado o excesso de óleo do bioplástico com um papel toalha, e, em seguida, este foi colocado em estufa a $105^{\circ} \mathrm{C}$ por $24 \mathrm{~h}$ para secagem e posterior pesagem a fim de obter a massa final. Por meio dessa técnica, é possível determinar a perda de massa do material por solubilização em óleo pela Equação 1, sendo os resultados expressos em porcentagem (Fakhouri et al., 2012). 
Solubilidade em Óleo $(\%)=\frac{(\text { Massa inicial }(\mathrm{g})-\operatorname{Massa} \text { final }(\mathrm{g})) \times 100}{\operatorname{Massa} \operatorname{inicial}(\mathrm{g})}$

A análise de gramatura e espessura seguiu a metodologia descrita por Almeida et al. (2013). Por fim, a medida de opacidade foi realizada com base na metodologia desenvolvida por Rocha et al. (2014), utilizando um espectrofotômetro (Shimadzu, UV-1800), com as amostras recortadas em retângulos e aderidas à parede interna da cubeta; foi utilizado o ar como controle $\mathrm{e}$ comprimento de onda em luz visível de $500 \mathrm{~nm}$ para cada filme.

A análise colorimétrica foi realizada com um espectrocolorímetro Color Quest XE (Hunter Lab) equipado com iluminante D65 e ângulo de observação de $10^{\circ}$, leitura no modo reflectância especular excluída por leitura direta em sistema CIELAB. Foram determinados os seguintes parâmetros: $L^{*}, a^{*}, b^{*}$, croma $\left(C^{*}{ }_{\mathrm{ab}}\right)$ e ângulo de tonalidade - hue (hab). Os valores de $C^{*}{ }_{\mathrm{ab}}$ e $\mathrm{h}_{\mathrm{ab}}$ foram calculados de acordo com Equações 2, 3 e 4, respectivamente.

$$
\begin{aligned}
& \mathrm{C}_{\mathrm{ab}}{ }=\sqrt{\left(\mathrm{a}^{*}\right)^{2}+\left(\mathrm{b}^{*}\right)^{2}} \\
& \mathrm{~h}_{\mathrm{ab}}=\arctan \frac{\mathrm{b}^{*}}{\mathrm{a}^{*}}, \text { para }+\mathrm{a}^{*},+\mathrm{b}^{*} \\
& \mathrm{~h}_{\mathrm{ab}}=180+\arctan \frac{\mathrm{b}^{*}}{\mathrm{a}^{*}}, \text { para }-\mathrm{a}^{*},-\mathrm{b}^{*}
\end{aligned}
$$

As propriedades térmicas dos bioplásticos foram avaliadas utilizando um calorímetro diferencial de varredura (Shimadzu, DSC60). As amostras foram pesadas em uma panela de alumínio, com massas de filme que variaram de 0,6 a $0,9 \mathrm{mg}$ e faixa de temperatura de 0 a $500{ }^{\circ} \mathrm{C}$. A taxa de aquecimento foi de $10^{\circ} \mathrm{C} / \mathrm{min}$ com argônio como gás de arraste.

Todos os resultados experimentais foram expressos em média \pm desvio-padrão. $\mathrm{O}$ conjunto de dados foram submetidos à análise de variância (ANOVA) e teste de Tukey com $5 \%$ de probabilidade $(p<0,05)$ pelo programa IBM-SPSS Statistics 23 .

\section{Resultados e discussão}

\subsection{Caracterização físico-química da farinha de subproduto de broto}

A caracterização físico-química da matéria-prima é essencial para compreender o comportamento do produto na formulação dos filmes elaborados. Os resultados das análises físico-químicas realizadas na farinha de subproduto de broto podem ser observados na Tabela 2.

Tabela 2. Propriedades físico-químicas da farinha de subproduto de broto seco (FSBS).

\begin{tabular}{lc}
\hline Propriedades & Farinha de subproduto de broto seco (FSBS) \\
\hline Umidade (\%) & $8,31 \pm 0,13$ \\
\hline Cinzas (\%) $)^{1}$ & $3,59 \pm 0,03$ \\
\hline Proteínas $(\%)^{1}$ & $2,58 \pm 0,12$ \\
\hline${\text { Lipídeos }(\%)^{1}}^{1}$ & $5,76 \pm 0,09$ \\
\hline Fibras (\%) & $47,02 \pm 0,30$ \\
\hline Carboidratos $(\%)^{1,2}$ & $41,60 \pm 0,24$ \\
\hline pH & $5,80 \pm 0,01$ \\
\hline Acidez & $0,64 \pm 0,04$ \\
\hline Atividade de água & $0,51 \pm 0,00$ \\
\hline Índice de absorção de água $(\mathrm{g} / \mathrm{g})$ & $3,88 \pm 0,11$ \\
\hline Índice de solubilidade em água $(\mathrm{g} / \mathrm{g})$ & $0,09 \pm 0,01$ \\
\hline Índice de absorção de óleo $(\mathrm{g} / \mathrm{g})$ & $1,97 \pm 0,46$ \\
\hline$L^{*}(l u m i n o s i d a d e)$ & $52,19 \pm 0,65$ \\
\hline
\end{tabular}


Tabela 2. Continuação...

\begin{tabular}{lc}
\hline Propriedades & Farinha de subproduto de broto seco (FSBS) \\
\hline$a^{*}$ (verde a vermelho) & $4,58 \pm 0,30$ \\
\hline$b^{*}$ (amarelo a azul) & $11,90 \pm 0,61$ \\
\hline $\mathrm{C}^{*}$ ab (saturação) & $12,76 \pm 0,52$ \\
\hline $\mathrm{h}_{\mathrm{ab}}$ (tonalidade) & $68,90 \pm 2,03$ \\
\hline${ }^{1}$ Resultados expressos em matéria seca: ${ }^{2} \mathrm{O}$ resultado de carboidrato foi obtido pelo método da diferença.
\end{tabular}

A farinha elaborada a partir de subprodutos da colheita de brotos apresentou umidade inferior a $10 \%$ e alto conteúdo de fibras $(47,02 \%)$. Badwaik et al. (2013) caracterizaram o broto de bambu e encontraram um valor $0,7 \mathrm{vez}$ menor no teor de fibras $(34,41 \%)$ e de lipídeos $(4,41 \%)$ e 13 vezes maior de proteínas $(34,51 \%)$, quando comparado à farinha de subproduto de brotos deste estudo. Brotos de soja, estudados por Vilas Boas et al. (2002), também apresentaram diferenças na composição centesimal quando comparados à farinha de subproduto de brotos elaborada, como um teor de fibras 12 vezes menor (3,93\%) e valores 3 vezes maior de lipídeos (18,85\%) e 14 vezes maior de proteínas $(35,84 \%)$.

As características da composição centesimal da farinha podem influenciar as propriedades tecnológicas apresentadas pelos filmes elaborados. Cazón et al. (2016) relatam diversos componentes que podem servir de base para formação de um bioplástico, como polissacarídeos (fibra, amido e pectina), lipídeos (ceras, acilgliceróis e ácidos graxos) e proteínas. Farinhas com alta quantidade de fibras obtidas de resíduos vegetais podem ser utilizadas originando diversos benefícios para os produtos da indústria de embalagens, por exemplo, aumentar as propriedades mecânicas e a estabilidade térmica e diminuir a adsorção de água (Crizel et al., 2018).

Sobre as demais análises realizadas na farinha de subproduto de broto, observou-se que o índice de absorção de água foi 20 vezes maior quando comparado ao índice de absorção de óleo, apontando que a farinha possui considerável retenção de água e baixa retenção de óleo. De acordo com Rosell et al. (2009), a capacidade de reter água é definida como a quantidade de água retida pela amostra sem sofrer qualquer estresse. A capacidade de ligação da água é o termo usado para o índice de absorção de água ou a quantidade de água retida pela fibra após ter sido submetida à centrifugação. O índice de absorção de óleo para farinhas vegetais depende do tamanho da partícula, da estrutura dos polissacarídeos, da fonte vegetal e das condições de produção da farinha. Além disso, é um parâmetro importante quando o produto passa por processo de extrusão.

Em relação à análise colorimétrica, a amostra apresentou $a^{*}$ de 4,58 $\pm 0,30$ e $b^{*}$ de $11,90 \pm 0,61$, indicando uma cor marrom-clara, que pode ser visualizada na Figura 1. Os parâmetros utilizados para quantificar e qualificar a cor, respectivamente, são $C_{\mathrm{ab}} * \mathrm{e} \mathrm{h}_{\mathrm{ab}}$. Um valor de $\mathrm{h}_{\mathrm{ab}}$ igual a $0^{\circ}$ corresponde a vermelho $\left(a^{*}\right), 90^{\circ}$, a amarelo $\left(b^{*}\right), 180^{\circ}$, a verde $\left(-a^{*}\right)$, e $270^{\circ}$, a azul $\left(-b^{*}\right)$. Como a farinha apresentou cor marrom-clara, os valores $C_{\mathrm{ab}} *$ foram semelhantes aos descritos para $b^{*}$. Essa coloração típica deve-se ao elevado conteúdo de sementes e folhas presentes na matéria-prima (Figura 1a). Além disso, pode-se observar que alguns grânulos de fibras, possivelmente provenientes das sementes não germinadas, permaneceram inteiros, aparentando um aspecto não uniforme nos bioplásticos desenvolvidos.

Como a água é o principal componente na maioria dos resíduos agroindustriais, as propriedades físico-químicas da água influenciam fortemente o comportamento físico-químico da farinha durante o armazenamento, antes da elaboração dos bioplásticos. A atividade de água (aw) da farinha de subproduto de brotos foi de 0,51 , o que representa uma baixa quantidade de água disponível para demais reações que possam comprometer a estabilidade da farinha.

Silva et al. (2019) também elaboraram farinha de subproduto de brotos a partir de uma doação da empresa Brottar (Viamão, RS, Brasil); no entanto, houve diferenças na proporção de brotos, contendo quantidades de brotos de feijão, trevo, amaranto, brócolis, rabanete e, 
predominantemente, alfafa. Esses autores encontraram valores de 8,88\% para umidade, 3,76\% para cinzas, 2,34\% para proteínas, 4,29\% para lipídeos, 37,19\% para carboidratos e 52,41\% para fibras. Ainda, os mesmos autores obtiveram valores de 3,96 para o índice de absorção de água, 2,56 para o índice de absorção de óleo e 0,07 para o índice de solubilidade em água. Sobre o pH, a acidez e a atividade de água, a farinha elaborada neste estudo apresentou valores de 6,50, 0,41 e 0,56, respectivamente. Por fim, sua análise colorimétrica indicou valores de 56,08 para $L^{*}, 4,58$ para $a^{*}$, 9,86 para $b^{*}, 10,87$ para $C_{\mathrm{ab}} *$ e 65,04 para $h_{\mathrm{ab}}$. Os valores encontrados por Silva et al. (2019) são muito próximos aos presentes neste estudo, incluindo compatibilidade de cor, indicando a possível reprodutibilidade dessa farinha independentemente da proporção desses resíduos de brotos.

\subsection{Propriedades tecnológicas do bioplástico}

Os resultados das propriedades tecnológicas dos filmes elaborados podem ser observados na Tabela 3. 
Tabela 3. Propriedades tecnológicas das diferentes formulações de bioplásticos elaborados.

\begin{tabular}{|c|c|c|c|c|c|c|}
\hline Propriedades & $\begin{array}{l}\text { Filmes com } 0 \% \text { de } \\
\text { FSBS com amido PA }\end{array}$ & $\begin{array}{c}\text { Filme com } 0 \% \text { de FSBS } \\
\text { (controle) }\end{array}$ & $\begin{array}{c}\text { Filme com } 2,5 \% \text { de } \\
\text { FSBS }\end{array}$ & Filme com $5 \%$ de FSBS & $\begin{array}{c}\text { Filme com } 7,5 \% \text { de } \\
\text { FSBS }\end{array}$ & $\begin{array}{c}\text { Filme com } 10 \% \text { de } \\
\text { FSBS }\end{array}$ \\
\hline Umidade (\%) & $16,70 \pm 1,39^{\mathrm{c}}$ & $24,46 \pm 1,31^{\mathrm{a}}$ & $21,41 \pm 1,19^{\mathrm{b}}$ & $20,46 \pm 1,25^{\mathrm{b}}$ & $21,01 \pm 0,35^{\mathrm{b}}$ & $20,71 \pm 0,66^{\mathrm{b}}$ \\
\hline Gramatura $\left(\mathrm{g} / \mathrm{cm}^{2}\right)$ & $0,0349 \pm 0,01^{\mathrm{a}}$ & $0,0211 \pm 0,01^{\mathrm{b}}$ & $0,0283 \pm 0,01^{\mathrm{ab}}$ & $0,0227 \pm 0,01^{\mathrm{b}}$ & $0,0233 \pm 0,01^{\mathrm{b}}$ & $0,0220 \pm 0,01^{\mathrm{b}}$ \\
\hline Espessura (mm) & $0,31 \pm 0,04^{\mathrm{a}}$ & $0,16 \pm 0,03^{\mathrm{c}}$ & $0,19 \pm 0,02^{\mathrm{bc}}$ & $0,20 \pm 0,01^{\mathrm{bc}}$ & $0,21 \pm 0,02^{\mathrm{bc}}$ & $0,23 \pm 0,02^{\mathrm{b}}$ \\
\hline Solubilidade em água (\%) & $19,12 \pm 1,98^{\mathrm{ab}}$ & $24,60 \pm 3,79^{\mathrm{a}}$ & $16,20 \pm 3,53^{b}$ & $15,18 \pm 1,65^{\mathrm{b}}$ & $14,07 \pm 5,54^{\mathrm{b}}$ & $13,67 \pm 4,69^{\mathrm{b}}$ \\
\hline Solubilidade em ácido (\%) & $45,67 \pm 28,02^{\mathrm{a}}$ & $55,02 \pm 2,67^{\mathrm{a}}$ & $45,43 \pm 8,79^{\mathrm{a}}$ & $50,30 \pm 14,14^{\mathrm{a}}$ & $65,57 \pm 8,17^{\mathrm{a}}$ & $67,35 \pm 2,79^{a}$ \\
\hline Solubilidade em óleo (\%) & $0,00 \pm 0,00^{\mathrm{a}}$ & $0,28 \pm 0,01^{\mathrm{a}}$ & $0,00 \pm 0,00^{\mathrm{a}}$ & $0,00 \pm 0,00^{\mathrm{a}}$ & $0,00 \pm 0,00^{\mathrm{a}}$ & $0,32 \pm 0,71^{\mathrm{a}}$ \\
\hline Opacidade & $\mathrm{Nd}$ & $2,36 \pm 0,17^{\mathrm{ab}}$ & $1,83 \pm 0,13^{\mathrm{c}}$ & $1,99 \pm 0,33^{b c}$ & $2,02 \pm 0,23^{\mathrm{bc}}$ & $2,50 \pm 0,25^{\mathrm{a}}$ \\
\hline$L^{*}$ & $39,16 \pm 1,63^{\mathrm{a}}$ & $38,57 \pm 0,54^{\mathrm{a}}$ & $38,66 \pm 0,72^{\mathrm{a}}$ & $36,03 \pm 1,06^{\mathrm{b}}$ & $34,04 \pm 0,46^{\mathrm{c}}$ & $32,98 \pm 0,56^{\mathrm{c}}$ \\
\hline$a^{*}$ & $-0,09 \pm 0,02^{\mathrm{d}}$ & $-0,03 \pm 0,04^{\mathrm{cd}}$ & $0,22 \pm 0,08^{\mathrm{c}}$ & $0,63 \pm 0,07^{\mathrm{b}}$ & $0,84 \pm 0,19^{\mathrm{b}}$ & $1,21 \pm 0,25^{\mathrm{a}}$ \\
\hline$b^{*}$ & $-0,16 \pm 0,12^{\mathrm{d}}$ & $-0,30 \pm 0,10^{\mathrm{d}}$ & $1,04 \pm 0,20^{\mathrm{c}}$ & $2,14 \pm 0,26^{\mathrm{b}}$ & $2,68 \pm 0,62^{\mathrm{ab}}$ & $3,41 \pm 0,57^{\mathrm{a}}$ \\
\hline$C_{\mathrm{ab}}^{*}$ & $0,20 \pm 0,11^{\mathrm{d}}$ & $0,30 \pm 0,09^{\mathrm{d}}$ & $1,06 \pm 0,21^{\mathrm{c}}$ & $2,23 \pm 0,27^{b}$ & $3,01 \pm 0,49^{\mathrm{a}}$ & $3,62 \pm 0,62^{\mathrm{a}}$ \\
\hline $\mathrm{h}_{\mathrm{ab}}$ & $232,83 \pm 19,45^{\mathrm{b}}$ & $259,97 \pm 12,78^{\mathrm{a}}$ & $78,23 \pm 3,02^{\mathrm{c}}$ & $73,43 \pm 1,15^{\mathrm{c}}$ & $73,04 \pm 6,61^{\mathrm{c}}$ & $70,55 \pm 1,06^{\mathrm{c}}$ \\
\hline
\end{tabular}

FSBS - Farinha de subproduto de broto seco; nd - não determinado; PA - pureza analítica; médias com letras iguais na horizontal não diferem entre si pelo teste de Tukey (5\%). 
Em relação à umidade, os filmes com adição de FSBS apresentaram valores similares de umidade sem diferença significativa entre eles, enquanto os filmes elaborados sem FSBS possuíram valores de umidade com diferença significativa quando comparados com as formulações com adição de FSBS. Um estudo realizado por Mihai et al. (2014) indicou que a adição de fibras pode diminuir a retenção de água dos materiais, o que pode explicar a interferência no teor de umidade dos bioplásticos. Sobre a espessura, não foi observado seu aumento significativo com a adição de FSBS, exceto na amostra de filme com 10\% de FSBS, que apresentou diferença significativa da amostra controle. A amostra de filme de amido PA teve um aumento de espessura de mais de 1,3 vez quando comparado às demais formulações estudadas. A espessura é uma variável que permite avaliar a homogeneidade dos filmes e interfere nas propriedades de barreira. As variações de espessura podem ocorrer pela falta de homogeneidade no processo de evaporação do solvente e dificuldade de espalhar a solução filmogênica na placa de Petri. Daudt et al. (2017), em filmes elaborados com farinha de semente de pinheiro brasileiro (Araucaria angustifolia), encontraram espessura entre 0,05 e $0,17 \mathrm{~mm}$, valores próximos aos obtidos neste trabalho.

A gramatura variou de 0,02 a $0,03 \mathrm{~g} / \mathrm{cm}^{2}$ entre os filmes elaborados. Henrique et al. (2008) elaboraram filmes biodegradáveis a partir de amidos modificados de mandioca e obtiveram valores de gramatura próximos aos encontrados neste estudo, variando de 0,02 a $0,05 \mathrm{~g} / \mathrm{cm}^{2}$. Os filmes que apresentam maiores gramaturas oferecem maiores resistências mecânicas, ou seja, maior resistência a deformações mecânicas ao bioplástico (Almeida et al., 2013; Henrique et al., 2008).

Em relação ao índice de solubilidade em água, as amostras apresentaram diminuição dos valores com a adição de FSBS. Essa queda da solubilidade pode ser explicada em razão de a capacidade de retenção de água da farinha de subproduto de broto adicionada ser inferior à capacidade de retenção de água do amido comercial utilizado. Sobre a solubilidade em ácido, os resultados apontados não tiveram uma tendência conforme o aumento de FSBS adicionada ou o tipo de amido (comercial ou PA), com valores entre $45 \%$ e $67 \%$. Amostras tiveram um resultado esperado por causa da interação com o pH baixo da solução de ácido clorídrico $1 \mathrm{~mol} / \mathrm{L}(0,2)$, interferindo fortemente na estabilidade do filme, como pode ser observado também no estudo de Fakhouri et al. (2012), no qual todas as misturas de filmes elaboradas apresentaram $100 \%$ de solubilidade em ácido. Percebe-se que a solubilidade em ácido é superior quando comparada à solubilidade em água. Por fim, em relação aos resultados de solubilidade em óleo, pode-se perceber que todos os bioplásticos elaborados não se mostraram influenciados pela presença do óleo vegetal, sofrendo alterações mínimas em seu peso após o tratamento.

A opacidade dos filmes variou entre as formulações estudadas. O controle e o filme com $10 \%$ de FSBS apresentaram os maiores valores de opacidade. Segundo um estudo realizado por Rocha et al. (2014), a opacidade pode ser influenciada pela proporção de glicerol e pelo pH da solução, o que poderia explicar a diferença obtida no presente estudo.

Sobre a análise colorimétrica, a luminosidade não teve uma tendência com o aumento da proporção de FSBS. Em relação aos parâmetros $a^{*}$ e $b^{*}$, pode-se observar que, à medida que ocorreu aumento da proporção de FSBS, os filmes foram adquirindo coloração amarronzada, próxima à FSBS. Como esperado, a amostra controle e a amostra de amido PA tiveram tonalidades mais claras e baixa intensidade de cor pela não inclusão da FSBS, enquanto os filmes com o aumento da proporção de FSBS, tonalidades mais escuras (valores em entre 70 a 78), quando comparados às amostras controle e com amido PA (valores de 259 e 232, respectivamente), além de aumento gradual da saturação conforme o crescimento da proporção de FSBS. Notou-se que os valores de tonalidade e saturação, com o aumento da proporção de FSBS, foram se aproximando 
do valor apresentado pela FSBS elaborada (de acordo com a Tabela 2). Ademais, o aspecto visual do filme com adição de FSBS, em qualquer proporção, acarretou ao produto aspecto singular e característico da FSBS elaborada - características colorimétricas observadas na Figura 1b.

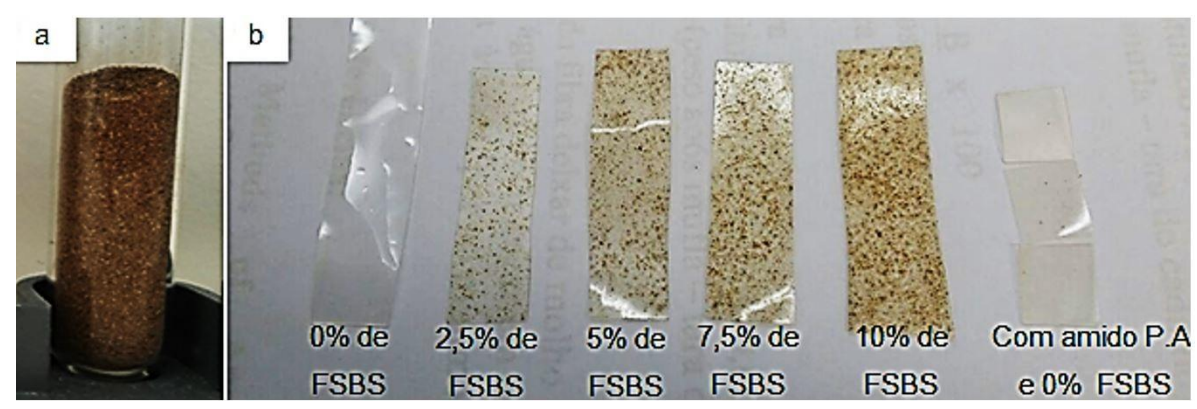

Figura 1. Coloração da farinha de subproduto de broto (a); coloração dos diferentes bioplásticos elaborados (FSBS - farinha de subproduto de broto seco; PA - pureza analítica) (b).

A estabilidade térmica é um determinante importante das propriedades funcionais. Isso ocorre porque a aplicação do calor altera os parâmetros estruturais que influenciam a funcionalidade e a estabilidade do bioplástico. As propriedades térmicas e termodinâmicas das amostras podem ser observadas na Tabela 4. Foi notado que os diferentes componentes e concentrações nos filmes possuíram distintas compatibilidades durante o aquecimento térmico. Para todas os filmes, três picos foram observados, sendo os dois primeiros endotérmicos - um de transição vítrea e outro de fusão - e o terceiro exotérmico, o que pode ser explicado pela pluralidade de ingredientes e suas reações à elevada temperatura. As temperaturas de transição vítrea e de fusão são informações relevantes para estimar propriedades de barreira ao oxigênio, vapor de água e medir as melhores condições de armazenagem e transporte, bem como para usos industriais. Nos resultados obtidos por DSC, não foram observadas mudanças significativas com o aumento da concentração de FSBS nas formulações dos filmes. 
Tabela 4. Propriedades térmicas e termodinâmicas das diferentes formulações de bioplásticos elaborados.

\begin{tabular}{|c|c|c|c|c|c|c|c|}
\hline Prop & es térmicas & $\begin{array}{c}\text { Filmes com } 0 \% \text { de } \\
\text { FSBS com amido PA } \\
\end{array}$ & $\begin{array}{l}\text { Filme com } 0 \% \text { de } \\
\text { FSBS (controle) } \\
\end{array}$ & $\begin{array}{c}\text { Filme com } 2,5 \% \text { de } \\
\text { FSBS } \\
\end{array}$ & $\begin{array}{c}\text { Filme com } 5 \% \text { de } \\
\text { FSBS } \\
\end{array}$ & $\begin{array}{c}\text { Filme com } 7,5 \% \text { de } \\
\text { FSBS } \\
\end{array}$ & $\begin{array}{c}\text { Filme com } 10 \% \text { de } \\
\text { FSBS } \\
\end{array}$ \\
\hline \multirow{4}{*}{$\mathrm{Tf}_{1}\left({ }^{\circ} \mathrm{C}\right)$} & To $\left({ }^{\circ} \mathrm{C}\right)$ & $56,59 \pm 10,46^{\mathrm{b}}$ & $50,32 \pm 2,79^{\mathrm{b}}$ & $114,65 \pm 10,26^{\mathrm{a}}$ & $116,00 \pm 12,52^{\mathrm{a}}$ & $90,77 \pm 19,84^{\mathrm{ab}}$ & $70,19 \pm 14,04^{\mathrm{b}}$ \\
\hline & $\operatorname{Tp}\left({ }^{\circ} \mathrm{C}\right)$ & $87,40 \pm 11,20^{\mathrm{b}}$ & $76,11 \pm 4,91^{\mathrm{b}}$ & $127,27 \pm 10,34^{\mathrm{a}}$ & $130,51 \pm 3,38^{\mathrm{a}}$ & $108,31 \pm 12,65^{\mathrm{ab}}$ & $105,43 \pm 17,17^{\mathrm{ab}}$ \\
\hline & $\mathrm{Tc}\left({ }^{\circ} \mathrm{C}\right)$ & $127,24 \pm 6,06^{\mathrm{a}}$ & $126,81 \pm 4,23^{\mathrm{a}}$ & $151,57 \pm 8,89^{\mathrm{a}}$ & $133,89 \pm 13,67^{\mathrm{a}}$ & $116,97 \pm 19,59^{\mathrm{a}}$ & $128,33 \pm 16,67^{\mathrm{a}}$ \\
\hline & $\Delta \mathrm{Hg}\left({\left.\mathrm{J} . \mathrm{g}^{-1}\right)}\right.$ & $-103,34 \pm 15,26^{\mathrm{c}}$ & $-122,54 \pm 7,77^{c}$ & $-20,97 \pm 13,49^{\mathrm{ab}}$ & $-23,90 \pm 3,74^{\mathrm{ab}}$ & $-15,01 \pm 12,66^{\mathrm{a}}$ & $-51,97 \pm 6,42^{\mathrm{b}}$ \\
\hline \multirow{4}{*}{$\mathrm{Tf}_{2}\left({ }^{\circ} \mathrm{C}\right)$} & To $\left({ }^{\circ} \mathrm{C}\right)$ & $286,20 \pm 0,73^{\mathrm{ab}}$ & $293,19 \pm 11,59^{\mathrm{a}}$ & $287,84 \pm 3,32^{\mathrm{ab}}$ & $293,38 \pm 6,26^{\mathrm{a}}$ & $289,13 \pm 5,08^{\mathrm{ab}}$ & $273,21 \pm 6,26^{b}$ \\
\hline & $\operatorname{Tp}\left({ }^{\circ} \mathrm{C}\right)$ & $292,32 \pm 5,56^{\mathrm{a}}$ & $301,73 \pm 18,45^{\mathrm{a}}$ & $291,84 \pm 2,66^{\mathrm{a}}$ & $298,29 \pm 7,32^{\mathrm{a}}$ & $293,91 \pm 2,85^{\mathrm{a}}$ & $278,20 \pm 5,84^{\mathrm{a}}$ \\
\hline & $\mathrm{Tc}\left({ }^{\circ} \mathrm{C}\right)$ & $295,55 \pm 0,28^{\mathrm{a}}$ & $301,00 \pm 9,23^{\mathrm{a}}$ & $303,26 \pm 8,36^{\mathrm{a}}$ & $307,18 \pm 11,42^{\mathrm{a}}$ & $302,11 \pm 3,78^{\mathrm{a}}$ & $287,85 \pm 4,20^{\mathrm{a}}$ \\
\hline & $\Delta \mathrm{Hg}\left({\left.\mathrm{J} . \mathrm{g}^{-1}\right)}\right.$ & $-21,72 \pm 7,81^{\mathrm{a}}$ & $-19,34 \pm 6,42^{\mathrm{a}}$ & $-21,97 \pm 16,61^{\mathrm{a}}$ & $-16,53 \pm 8,44^{\mathrm{a}}$ & $-17,43 \pm 4,45^{\mathrm{a}}$ & $-12,48 \pm 1,49^{\mathrm{a}}$ \\
\hline \multirow{4}{*}{$\mathrm{Tf}_{3}\left({ }^{\circ} \mathrm{C}\right)$} & To $\left({ }^{\circ} \mathrm{C}\right)$ & $346,53 \pm 4,66^{\mathrm{a}}$ & $352,30 \pm 7,65^{\mathrm{a}}$ & $352,02 \pm 12,06^{\mathrm{a}}$ & $353,47 \pm 6,36^{\mathrm{a}}$ & $347,99 \pm 6,91^{\mathrm{a}}$ & $344,37 \pm 18,81^{\mathrm{a}}$ \\
\hline & $\operatorname{Tp}\left({ }^{\circ} \mathrm{C}\right)$ & $362,88 \pm 0,88^{\mathrm{a}}$ & $360,52 \pm 5,47^{\mathrm{a}}$ & $358,46 \pm 5,11^{\mathrm{a}}$ & $369,21 \pm 7,36^{\mathrm{a}}$ & $365,85 \pm 7,95^{\mathrm{a}}$ & $365,96 \pm 4,38^{\mathrm{a}}$ \\
\hline & $\mathrm{Tc}\left({ }^{\circ} \mathrm{C}\right)$ & $364,38 \pm 9,41^{\mathrm{a}}$ & $371,73 \pm 8,51^{\mathrm{a}}$ & $374,51 \pm 1,07^{\mathrm{a}}$ & $382,67 \pm 4,52^{\mathrm{a}}$ & $384,12 \pm 9,03^{\mathrm{a}}$ & $383,25 \pm 5,74^{\mathrm{a}}$ \\
\hline & $\Delta \mathrm{Hg}\left({\left.\mathrm{J} . \mathrm{g}^{-1}\right)}\right.$ & $33,77 \pm 9,76^{\mathrm{b}}$ & $56,93 \pm 14,49^{\mathrm{ab}}$ & $110,08 \pm 36,48^{\mathrm{a}}$ & $103,87 \pm 14,85^{\mathrm{ab}}$ & $101,69 \pm 18,30^{\text {ab }}$ & $69,45 \pm 35,91^{\mathrm{ab}}$ \\
\hline
\end{tabular}

FSBS - farinha de subproduto de broto seco; $\mathrm{Tf}_{1}$ - temperatura de fusão do pico $1 ; \mathrm{Tf}_{2}$ - temperatura de fusão do pico $2 ; \mathrm{Tf}_{3}$ - temperatura de fusão do pico $3 ; \mathrm{To}$ - temperatura inicial; $\mathrm{Tp}$ - temperatura do pico; $\mathrm{Tc}$ - temperatura final; $\Delta \mathrm{Hg}$ - entalpia; PA - pureza analítica; Médias com letras iguais na horizontal não diferem entre si pelo teste de Tukey (5\%). 
Portanto, a farinha de subproduto de broto se mostrou eficiente para aplicação em bioplásticos, e, após as análises, pode-se sugerir que seu uso seja destinado a produtos mais oleosos, no entanto não é recomendada para alimentos com elevada umidade e acidez por causa do alto valor de solubilidade nesses solventes. O bioplástico elaborado também pode ser destinado a alimentos que não sofram processamento térmico a elevadas temperaturas, visto a temperatura relativamente baixa de transição e a modificação de seus componentes. Além disso, a aparência do bioplástico mostrou se adequar às tendências do mercado de embalagens alternativas e biodegradáveis.

\section{Conclusões}

$\mathrm{O}$ subproduto de broto se mostrou um resíduo agroindustrial adequado à produção de farinha para aplicação no desenvolvimento de bioplástico. A farinha elaborada apresentou uma composição físico-química com possível potencial para aplicação em bioplásticos, principalmente por causa de seu elevado conteúdo de fibras totais. A farinha de subproduto de broto exibiu alta capacidade de retenção de água e baixa retenção de óleo. A cor da farinha influenciou diretamente a cor dos filmes com o aumento da proporção de FSBS. Sobre os bioplásticos formulados, o aumento da proporção de farinha de subproduto de brotos proporcionou aumento na opacidade, porém não interferiu na gramatura nem nos valores de solubilidade em ácido. Os filmes biodegradáveis elaborados com farinha de subproduto de broto são adequados a embalar ou revestir produtos com baixo teor de umidade e baixa acidez, além de alimentos com elevado teor de gordura. No entanto, novos estudos devem ser realizados para caracterizar outras propriedades mecânicas e físicas e otimizar a formulação testada, bem como sua aplicabilidade na indústria de embalagens.

\section{Agradecimentos}

Ao Conselho Nacional de Desenvolvimento Científico e Tecnológico (CNPq), pela bolsa PIBIC concedida; à estrutura da Universidade Federal de Ciências da Saúde de Porto Alegre (UFCSPA); à empresa Brottar, pelo fornecimento dos resíduos de brotos; e ao Laboratório de Tecnologia e Processamento de Alimentos (LATEPA), laboratório integrante do Departamento de Engenharia Química (DEQUI) da Universidade Federal do Rio Grande do Sul (UFRGS).

\section{Referências}

Albuquerque, P. B. S., \& Malafaia, C. B. (2018). Perspectives on the production, structural characteristics and potential applications of bioplastics derived from polyhydroxyalkanoates. International Journal of Biological Macromolecules, 107(Pt A), 615-625. PMid:28916381. http://dx.doi.org/10.1016/j.ijbiomac.2017.09.026

Almeida, D. A., Woiciechowski, A. L., Wosiacki, G., Prestes, R. A., \& Pinheiro, L. A. (2013). Propriedades físicas, químicas e de barreira em filme formados por blenda de celulose bacteriana e fécula de batata. Polímeros, Ciência e Tecnologia, 23(4), 538-546.

Association of Official Analytical Chemists - AOAC. (1997). Official methods of analysis (Vol. 2, 16th ed.). Washington: AOAC.

Badwaik, L. S., Borah, P. K., \& Deka, S. C. (2013). Antimicrobial and enzymatic antibrowning film used as coating for bamboo shoot quality improvement. Carbohydrate Polymers, 103, 213-220. PMid:24528722.

Barros, T. T., Tosi, M. M., \& Assis, O. B. G. (2017). Aproveitamento de rejeitos da cadeia hortifrutícula no processamento de plásticos biodegradáveis. Revista Gestão Industrial, 13(2), 215-229.

Cazón, P., Velazquez, G., Ramírez, J. A., \& Vázquez, M. (2017). Polysaccharide-based films and coatings for food packaging: A review. Food Hydrocolloids, 68, 136-148

Crizel, T. M., Rios, A. O., Alves, V. D., Bandarra, N., Moldão-Martins, M., \& Flôres, S. H. (2018). Biodegradable films based on gelatin and papaya peel microparticles with antioxidant properties. Food and Bioprocess Technology, 11(3), 536-550. http://dx.doi.org/10.1007/s11947-017-2030-0

Daudt, R. M., Sinrod, A. J. G., Avena-Bustillos, R. J., Kulkamp-Guerreiro, I. C., Marczak, L. D. F., \& Mchugh, T. H. (2017). Development of edible films based on Brazilian pine seed (Araucaria angustifolia) flour reinforced with husk powder. Food Hydrocolloids, 71, 60-67. http://dx.doi.org/10.1016/j.foodhyd.2017.04.033 
Fakhouri, F. M., Fontes, L. C. B., Gonçalves, P. V. M., Milanez, C. R., Steel, C. J., \& Collares-Queiroz, F. P. (2007). Filmes e coberturas comestíveis composta à base de amidos nativos e gelatina na conservação e aceitação sensorial de uvas Crimson. Food Science and Technology (Campinas), 27(2), 369-375. http://dx.doi.org/10.1590/S0101-20612007000200027

Fakhouri, F. M., Martelli, S. M., Bertan, L. C., Yamashita, F., Mei, L. H. I., \& Queiroz, F. P. C. (2012). Edible films made from blend of manioc starch and gelatin - Influence of different types of plasticizer and different levels of macromolecules on their properties. LWT, 49(1), 149-154. http://dx.doi.org/10.1016/j.Iwt.2012.04.017.

Gomes, L. P., Souza, H. K. S., Campiña, J. M., Andrade, C. T., Silva, A. F., Gonçalves, M. P., \& Paschoalin, V. M. F. (2019). Edible chitosan films and their nanosized counterparts exhibit antimicrobial activity and enhanced mechanical and barrier properties. Molecules (Basel, Switzerland), 24(1), 1-14. PMid:30602680.

Guillon, F., \& Champ, M. (2000). Structural and physical properties of dietary fibres, and consequences of processing on human physiology. Food Research International, 33(3-4), 233-245. http://dx.doi.org/10.1016/S0963-9969(00)00038-7

Henrique, C. M., Cereda, M. P., \& Sarmento, S. B. S. (2008). Características físicas de filmes biodegradáveis produzidos a partir de amidos modificados de mandioca. Food Science and Technology (Campinas), 28(1), 231-240. http://dx.doi.org/10.1590/S0101-20612008000100033

Instituto Adolfo Lutz - IAL. (2008). Normas analíticas do Instituto Adolfo Lutz: Métodos físico-químicos para análises de alimentos (4. ed., 1020 p.). São Paulo: IAL.

Karan, H., Funk, C., Grabert, M., Oey, M., \& Hankamer, B. (2019). Green bioplastics as part of a circular bioeconomy. Trends in Plant Science, 24(3), 237-249. PMid:30612789.

http://dx.doi.org/10.1016/j.tplants.2018.11.010

Lopez, G., Artetxe, M., Amutio, M., Alvarez, J., Bilbao, J., \& Olazar, M. (2018). Recent advances in the gasification of waste plastics. A critical overview. Renewable \& Sustainable Energy Reviews, 82, 576-596.

http://dx.doi.org/10.1016/j.rser.2017.09.032

Lubis, M., Harahap, M. B., Ginting, M. H. S., Sartika, M., \& Azmi, H. (2018). Production of bioplastic from avocado seed starch reinforced with microcrystalline cellulose from sugar palm fibers. Journal of Engineering Science and Technology, 13(2), 381-393.

Melo, P. T. S., Aouada, F. A., \& Moura, M. R. (2017). Fabricação de filmes bionanocompósitos à base de pectina e polpa de cacau com potencial uso como embalagem para alimentos. Quimica Nova, 4(3), 247-251. http://dx.doi.org/10.21577/0100-4042.20160188.

Mihai, M., Legros, N., \& Alemdar, A. (2014). Formulation-properties versatility of wood fiber biocomposites based on polylactide and polylactide/thermoplastic starch blends. Polymer Engineering and Science, 54(6), 1325-1340. http://dx.doi.org/10.1002/pen.23681

Moro, T. M. A., Ascheri, J. L. R., Ortiz, J. A. R., Carvalho, C. W. P., \& Meléndez-Arévalo, A. (2017). Bioplastics of native starches reinforced with passion fruit peel. Food and Bioprocess Technology, 10(10), 1798-1808. http://dx.doi.org/10.1007/s11947-017-1944-x

Oluwasina, O. O., Olaleye, F. K., Olusegun, S. J., Oluwasina, O. O., \& Mohallem, N. D. S. (2019). Influence of oxidized starch on physico mechanical, thermal properties, and atomic force micrographs of cassava starch bioplastic film. International Journal of Biological Macromolecules, 135, 282-293. PMid:31128189. http://dx.doi.org/10.1016/j.ijbiomac.2019.05.150

Robertson, J. A., Monredon, F. D., Dysseler, P., Guillon, F., Amado, R., \& Thibault, J. (2000). Hydration properties of dietary fibre and resistant starch: a European collaborative study. Lebensmittel-Wissenschaft + Technologie, 33(2), 72-79. http://dx.doi.org/10.1006/fstl.1999.0595

Rocha, G. O., Farias, M. G., Carvalho, C. W. P., Ascheri, J. L. R., \& Galdeano, M. C. (2014). Filmes compostos biodegradáveis a base de amido de mandioca e proteína de soja. Polímeros, 24(5), 587-595. http://dx.doi.org/10.1590/0104-1428.1355

Rosell, C. M., Santos, E., \& Collar, C. (2009). Physico-chemical properties of commercial fibres from different sources: A comparative approach. Food Research International, 42(1), 176-184.

http://dx.doi.org/10.1016/j.foodres.2008.10.003

Santana, R. F., Bonomo, R. C. F., Gandolfi, O. R. R., Rodrigues, L. B., Santos, L. S., Pires, A. C. S., Oliveira, C. P., Fontan, R. C. I., \& Veloso, C. M. (2018). Characterization of starch-based bioplastics from jackfruit seed plasticized with glycerol. Journal of Food Science and Technology, 55(1), 278-286. PMid:29358820. http://dx.doi.org/10.1007/s13197-017-2936-6

Silva, M. L. T., Brinques, G. B., \& Gurak, P. G. (2019). Utilização de farinha de subproduto de brotos para elaboração de massa alimentícia fresca. Brazilian Journal of Food Technology, 22(e2018063), e2018063. http://dx.doi.org/10.1590/1981-6723.06318

Vilas Boas, E. V. B., Barcelos, M. F. P., \& Lima, M. A. C. (2002). Tempo de germinação e características físicas, químicas e sensoriais dos brotos de soja e de milho nas formas isoladas e combinadas. Ciência e Agrotecnologia, 26(1), 148-156.

Financiamento: Nenhum. 